\title{
Caracterização e concepções de ensino-aprendizagem do corpo docente de um curso de medicina em desenvolvimento
}

\author{
Characterization and conceptions of teaching-learning from the faculty of a medicine \\ course in development
}
Caracterización y concepción de la enseñanza-aprendizaje del cuerpo docente de un curso de medicina en desarrollo

Karen Regina Santana1*, Raquel Rangel Cesario

\begin{abstract}
RESUMO
Objetivo: Caracterizar o corpo docente de um curso de medicina e suas concepções de ensino-aprendizagem em relação ao preconizado pelas Diretrizes Curriculares Nacionais (DCN). Métodos: Considerando que apenas a aprovação e a publicação das DCN não garantem sua implementação e que possa haver resistências às práticas inovadoras de ensino, este trabalho aplicou aos docentes o questionário COLT, através do qual obteve-se as concepções conscientes e inconscientes de aprendizado e ensino dos entrevistados no que se refere a centralização do professor, apreciação de metodologias ativas e orientação para a prática profissional. Resultados: O corpo docente é majoritariamente composto por mulheres, médicas, com pós-graduação e dedicação de tempo entre docência e outras atividades laborais. Os resultados permitem recomendar que os professores se envolvam mais com o desenvolvimento docente, na missão de formar profissionais que contribuam para a reorientação do sistema de saúde. Conclusão: O corpo docente pesquisado apresenta concepções de ensino-aprendizagem relativas à centralização do professor, apreciação de metodologias ativas e orientação para prática profissional adequadas ao perfil exigido pelas DCN, embora ainda sejam necessárias mais capacitações para torná-lo mais agente de mudança conceitual.
\end{abstract}

Palavras-chave: Guias como assunto, Educação médica, Ensino, Aprendizagem baseada em problemas, Docente de medicina.

\begin{abstract}
Objective: This work aims to characterize the faculty of a medical course and their teaching-learning concepts in relation to the recommended by the Curriculum Guidelines (CG). Methods: Considering that the approval and publication of the new curricular guidelines do not guarantee its implementation and the new teaching practices could find resistance to be applied, teachers were given the COLT questionnaire, through which the respondents' conscious and unconscious conceptions of learning and teaching were obtained with regard to teacher centredness, appreciation of active learning and orientation to professional practice. Results: Ours data showed that faculty was mainly composed of women, medical doctors, graduate degrees and dedication of time between teaching and other work activities. The results allow us to recommend the professors to be more involved with teacher development, in the mission of training professionals who contribute to the reorientation of the health system. Conclusion: The faculty presents teaching-learning concepts related to teaching centredness, appreciation of active learning and orientation to professional practice appropriate to the profile required by the CG, although more training is still needed to make the faculty more agent of conceptual change.
\end{abstract}

Key words: Guidelines as topic, Medical education, Teaching methodologies, Problem-based learning, Medical faculty.

${ }^{1}$ Centro Universitário Municipal de Franca (Uni-FACEF), Franca - SP.

*E-mail: profkarensantana@gmail.com

SUBMETIDO EM: 7/2020

ACEITO EM: 8/2020

PUBLICADO EM: 10/2020 


\section{RESUMEN}

Objetivo: Caracterizar el cuerpo docente de un curso de medicina y sus concepciones de enseñanzaaprendizaje, en relación al recomendado por las Directrices Curriculares Nacionales (DCN). Métodos: Considerando que solo la aprobación y la publicación de las DCN no garantizan su implementación y que se pueda haber resistencia a las prácticas innovadoras de la enseñanza, a los docentes fue aplicado el examen COLT, mediante de ello, se obtuvo las concepciones conscientes e inconscientes de aprendizaje y enseñanza a los entrevistados en lo que se refiere a la centralización del profesor, apreciación de metodologías activas y orientación para la práctica profesional. Resultados: El personal docente está compuesto principalmente por mujeres, médicos, con títulos de posgrado y dedicación de tiempo entre la enseñanza y otras actividades laborales. Los resultados nos permiten recomendar que los profesores se involucren más con el desarrollo docente, en la misión de formar profesionales que contribuyan para la reorientación del sistema de salud. Conclusión: El personal docente presenta concepciones de enseñanza-aprendizaje relacionados con la centralización del profesor, apreciación de metodologías activas y orientación para la práctica profesional adecuada al perfil requerido por las DCN, aunque todavía sean necesarias más capacitaciones para convertir el cuerpo docente más agente de mudanza conceptual.

Palabras clave: Guías como asunto, Educación médica, Enseñanza, Aprendizaje basado en problemas, Docente médico.

\section{INTRODUÇÃO}

Desde sua criação, o Sistema Único de Saúde (SUS) passa por processo de constante construção e avaliação, ainda carecendo de plena implantação de seus princípios. Nota-se baixa capacidade dos profissionais que adentram suas portas para resolver problemas de saúde da população.

Reconhecendo a grande influência das instituições de ensino na organização dos serviços de saúde, por interferirem na formação dos sujeitos que atuarão no sistema de saúde, as novas Diretrizes Curriculares Nacionais de Graduação em Medicina (DCN) propõem uma nova realidade educacional, apontando para a formação de um profissional que tenha responsabilidade social e compromisso com a cidadania, além de promover a saúde integral dos cidadãos (GOMES AP e REGO S, 2011; BRASIL, 2014; SILVA VO e SANTANA PMMA, 2015; CARABETTA JV, 2016).

Para essa nova realidade educacional, que se dá em contexto de transição de paradigmas, é preciso haver mudança de postura na relação professor-conhecimento-aluno, através do desenvolvimento de competências e habilidades que tornem a aprendizagem de fato significativa e não apenas uma transmissão rápida de conhecimento centrada na figura do professor (CARABETTA JV, 2016; CESARIO RR, et al, 2019).

O SUS demanda docentes que fomentem a humanização durante a graduação, bem como a reflexão e ação com responsabilidade social e compromisso com a cidadania, para que ela passe a ser a prática do educando quando esse se tornar um profissional e possa agir como promotor da saúde integral do ser humano.

Num passado recente a qualificação do professor em relação ao conteúdo específico de sua disciplina era considerada suficiente para o exercício da docência. Isso culminou em um contexto educacional com falta de habilidades para o ensino de profissionalização docente.

O critério de contratação dos docentes nos cursos médicos centrava-se na qualidade do desempenho do profissional médico em sua área técnica de atuação, porém, agora, percebe-se que a atividade docente demanda capacitação específica.

Hoje, o professor de medicina, na maioria das vezes médico, provavelmente formado pelo modelo hospitalocêntrico, deve atuar como educador na nova proposta de formar médicos, aliando o conhecimento técnico ao fazer pedagógico. Portanto, para reorientação da educação médica é necessário reformar os educadores pois não há como pensar em mudanças pedagógicas dissociadas do docente (PERIM GL, 2009; COSTA NMSC, 2010; GARCIA MAA e SILVA ANB, 2011). 
Nos últimos anos muito se tem discutido sobre metodologias de ensino centradas no aluno, nas quais almeja-se que ele assuma o lugar de protagonismo no cenário do aprendizado. Assim, na medida em que essas proposições avançam a passos largos, faz-se necessária a discussão acerca da capacitação do docente e seu impacto na promoção do ensino.

A concepção dos docentes no tocante ao ensino é atrelada de maneira consciente e inconsciente pelas suas concepções de aprendizado e ensino. Dessa maneira, o modo como os professores abordam o ensino está ligado à maneira como eles veem o ensino e às suas experiências de vida, como por exemplo sua formação acadêmica prévia. Isto posto, supõe-se que as diferentes abordagens de ensino dos docentes afetam diretamente as abordagens de aprendizagem dos alunos e, portanto, o desempenho acadêmico deles. Conclui-se que o desenvolvimento do corpo docente é peça fundamental no processo do aprendizado (JACOBS JC et al, 2012).

O diagnóstico prévio do perfil dos professores é pré-requisito para capacitação dos mesmos, pois tal processo exige mais do que o desenvolvimento das habilidades de ensino. Nesse sentido, objetiva-se caracterizar o corpo docente de um curso de medicina e suas concepções de ensino-aprendizagem em relação ao preconizado pelas DCN. Entendemos que a compreensão do perfil dos professores pode ser útil para os próprios, para atividades de desenvolvimento docente e para Institutos e departamentos, especialmente se envolvidos em uma reforma curricular em direção à educação centrada no aluno.

\section{MÉTODOS}

Apresenta-se um recorte de estudo exploratório quantitativo que visou mapear as concepções de aprendizagem e ensino dos corpos diretivo e docente de um curso de Medicina no interior de São Paulo, analisando sua adequação às DCN e ao projeto político-pedagógico (PPP) do curso, inaugurado em 2015. O curso médico em questão tem seu PPP embasado em metodologias ativas de aprendizagem, em especial a Aprendizagem Baseada em Problemas e a Metodologia da Problematização, e fomenta uma formação de médicos integrada às necessidades sociais, individuais e coletivas, a partir do reconhecimento e da vivência cotidiana do estudante com suas responsabilidades e atribuições no campo prático da saúde (BERBEL NN, 1998).

O curso é desenvolvido em 12 semestres, divididos em três ciclos consecutivos de aprendizagem, cada um com duração de quatro semestres. As unidades curriculares até o oitavo semestre são: Interação em Saúde na Comunidade (IESC); Tutoria; Laboratório Morfofuncional e Habilidades técnicas. Do nono até o $12^{\circ}$ semestre, Internato. O sujeito da pesquisa foram os 94 docentes do curso de Medicina no ano de 2019.

Para mensuração das concepções de ensino-aprendizagem dos professores utilizou-se o questionário Conceptions of learning and teaching (COLT), que permite obter as concepções conscientes e inconscientes de aprendizado e ensino dos entrevistados (JACOBS JC, et al., 2014).

O questionário consiste em 18 perguntas, com tempo de preenchimento de até 10 minutos. Para cada afirmativa, seis categorias de reação do entrevistado conforme suas concepções: 1 - Discordo Totalmente; 2 Discordo; 3- Neutro; 4- Concordo; 5- Concordo fortemente; 6- Não tenho opinião. Ao final, o respondente será avaliado com uma nota numérica (que vai de 1 até 5) em três escalas: "Centralização do professor", "Apreciação das metodologias ativas" e "Orientação para a prática profissional".

"Centralização do professor" reflete o ensino centrado no professor, focado na transmissão de conhecimentos em oposição ao perfil centrado no aluno, focado na mudança conceitual dos estudantes. Já os aspectos "Apreciação das metodologias ativas" e "orientação para a prática profissional" falam a respeito da afinidade do docente e orientação da sua prática pedagógica para, respectivamente, o uso de metodologias ativas de ensino-aprendizagem e orientação para a prática profissional.

Para cada uma dessas características, ou escalas, o teste emite cinco possíveis perfis, a saber: Transmissor (T), Organizador (O), Intermediário (I), Facilitador (F) e Agente de mudança conceitual (A), identificados aqui como uma gradação positiva de adequação para a prática docente em acordo com as diretrizes curriculares para graduação em Medicina estabelecidas no Brasil. Dessa maneira, ao procurar identificar os agentes de mudanças conceituais, o questionário dialoga com o proposto pelas DCN's. 
O projeto desta pesquisa foi submetido previamente ao Comitê de Ética em Pesquisa com Seres Humanos (CAAE 19903519.4.0000.5384) e aprovado pelo Parecer Consubstanciado no. 3.564.164. Os participantes receberam e assinaram Termos de Consentimento Livre e Esclarecido (TCLE) e todo o ordenamento ético e legal exigido pelo país foi seguido. Os dados sofreram análise descritiva com auxílio do software estatísticos Minitab.

\section{RESULTADOS}

A versão em português desse questionário estruturado foi entregue aos professores elegíveis e aplicada manualmente, por meio impresso anexado juntamente com a cópia do TCLE. O processo de convite para participar do estudo deu-se em encontros pessoais, um a um, durante atividades acadêmicas regulares no início do mês de dezembro de 2019. Com tal abordagem, foi possível acessar 74 docentes, dos 94 elegíveis. Dos 74 docentes aos quais foi entregue o questionário, 69 o devolveram respondido, sendo este o número total da amostra. Dos vinte docentes aos quais o questionário não foi entregue, dois foram desligados da instituição, duas estavam de licença maternidade e aos 16 restantes não tivemos acesso.

O questionário aplicado possui duas partes: na primeira, correspondente à identificação do participante, foram solicitados dados como sexo, idade, curso de graduação e modelo pedagógico através do qual se graduou, titulação máxima, carga horária semanal média na docência e unidades curriculares em que atua. A segunda parte consistiu em responder às 18 perguntas fechadas do COLT. Após a coleta os dados foram inseridos no sítio da Internet disponibilizado pelos autores do instrumento e sua interpretação recebida por correio eletrônico.

Sabendo-se que a língua, como todo sistema complexo, está sempre em transformação, para facilitar ainda mais o entendimento dos respondentes, à escala de Likert adicionou-se figuras denominadas "emojis". Essas figuras, geradas pelo sistema Unicode para representar emoções, como afirma Porter C (2014), "tornaram-se uma língua franca para determinados usuários de mensagens de texto e mídias sociais" (PAIVA VLMO, 2016).

\section{Caracterização demográfica}

O maior contingente dos participantes deste estudo é do sexo feminino $(55,07 \%)$. A maior parte é formada por profissionais médicos $(73,91 \%)$ sendo que os não médicos compreendem principalmente enfermeiros e farmacêuticos, entre psicólogos, nutricionista, médicos veterinários, biomédicos etc. No que tange à titulação, a maior parte dos respondentes são especialistas $(44,93 \%)$, seguidos de mestres $(31,88 \%)$ e doutores (23,19\%). Relativamente à idade, $51,72 \%$ dos inquiridos têm idade entre 29 e 45 anos (Tabela 1).

Tabela 1 - Distribuição por faixa etária

\begin{tabular}{ccc}
\hline Categorias & Frequência $^{*}$ & Percentual \\
\hline 29 a 35 anos & 13 & $22,41 \%$ \\
36 a 40 anos & 12 & $20,69 \%$ \\
41 a 45 anos & 5 & $8,62 \%$ \\
46 a 50 anos & 11 & $18,97 \%$ \\
51 a 55 anos & 6 & $10,34 \%$ \\
55 a 60 anos & 5 & $8,62 \%$ \\
Acima de 60 anos & 6 & $10,34 \%$ \\
\hline Total & $\mathbf{5 8}$ & $\mathbf{1 0 0} \%$ \\
\hline
\end{tabular}

Legenda: *11 dados faltantes. Fonte: Santana KR e Cesario RR, 2020.

No que diz respeito à carga horária semanal como docentes, $26,47 \%$ referiram se dedicar à atuação docente entre 4 e 10 horas semanais, o que corresponde a meio ou um dia na semana, e foram categorizados como baixa carga horária. Aqueles que se dedicam à docência por mais de um e até três dias da semana foram considerados com carga horária média e corresponderam a $54,41 \%$ da amostra. Por último, os que 
disseram dedicar mais de 31 horas semanais, ou o equivalente a mais de 4 dias na semana, foram considerados com carga horária alta e representam $19,12 \%$ da amostra. Tais informações nos permitem afirmar que $73,53 \%$ da amostra possui carga horária semanal como docente média ou alta.

Estratificando por profissão, os profissionais médicos respondentes possuem uma carga horária semanal média como docente de 18,1 horas por semana, valor menor do que a média dos respondentes não médicos, que foi de 26,8 horas por semana (Tabela 2).

Tabela 2 - Carga Horária Semanal na condição de docente

\begin{tabular}{ccc}
\hline Categorias & Frequência & Percentual \\
\hline Baixa (até 10h) & 18 & $26,47 \%$ \\
Média (de 11 a 30h) & 37 & $54,41 \%$ \\
Alta (> 31 h) & 13 & $19,12 \%$ \\
\hline Total & $\mathbf{6 8}$ & $\mathbf{1 0 0} \%$ \\
\hline
\end{tabular}

Fonte: Santana KR e Cesario RR, 2020.

Aproximadamente $90 \%$ dos docentes médicos foram formados pelo método pedagógico tradicional, restando apenas sete docentes médicos formados pelo método conhecido como PBL. Observou-se também que os docentes médicos formados pelo sistema "tradicional" têm idade média de 45,4 anos, enquanto os docentes formados pelo "PBL" têm idade média de 38,9 anos, e que mais de $50 \%$ dos docentes médicos possuem título de mestre ou doutor.

Quanto às unidades curriculares (UC) em que o entrevistado atua, pôde-se observar que 29,91\% responderam atuar em Habilidades, sendo essa a UC com a maior frequência absoluta. Tal resultado se justifica porque a UC Habilidades contém Habilidades de Comunicação, Habilidades de Informática e Habilidades Médicas em geral, com conteúdo de semiologia geral e dos diversos sistemas, Raciocínio Clínico e Habilidades Cirúrgicas. Importante observar que alguns professores estão envolvidos em mais de uma Unidade Curricular, o que faz o valor total da Tabela abaixo ultrapassar os 69 respondentes (Tabela 3). Apenas seis participantes $(5,13 \%)$ respondem pela gestão do curso, seja em atividades ligadas à reitoria da Instituição, seja professores do curso que compõem o Núcleo Docente Estruturante (NDE).

Tabela 3 - Docentes por vinculação a Unidades Curriculares

\begin{tabular}{ccc}
\hline Categorias & Frequência & Percentual \\
\hline Conhecimentos Gerais & 2 & $1,71 \%$ \\
Direção, Gestão, NDE & 6 & $5,13 \%$ \\
Habilidades & 35 & $29,91 \%$ \\
IESC & 15 & $12,82 \%$ \\
Internato & 25 & $21,37 \%$ \\
Laboratório & 11 & $9,40 \%$ \\
Tutoria & 23 & $19,66 \%$ \\
\hline Total & $\mathbf{1 1 7}$ & $\mathbf{1 0 0} \%$ \\
\hline
\end{tabular}

Fonte: Santana KR e Cesario RR, 2020.

Os resultados se assemelham ao encontrado em professores de um curso de Medicina no interior de Minas Gerais, onde 64\% dos professores são médicos, formados em sua maioria nas décadas de 1990 e 2000, o que corresponde à mesma faixa etária do nosso corpo docente. Como em nossa amostra, a maioria dos professores com pós-graduação strictu sensu e maior dedicação ao ensino que à profissão original estão no ciclo básico e são não-médicos (LEITE VT, et al., 2020). 


\section{Caracterização do perfil docente}

O perfil docente foi caracterizado segundo as três escalas propostas pelo COLT, tratando suas variáveis como sendo ordinais, devido ao entendimento de que a proposta das DCN é sair de uma metodologia de ensino-aprendizagem onde a transmissão do conhecimento ocorre de forma fechada, fragmentada, reprodutiva e desconexa da realidade e passar para uma metodologia libertadora focada no aluno, onde ele é protagonista do seu aprendizado, voltado para reflexão e para humanização. Assim, os perfis foram descritos em ordem de adequação às $\mathrm{DCN}$ do menos ao mais adequado respectivamente: transmissor $(\mathrm{T})$, organizador (O), intermediário (I), facilitador $(F)$ e agente de mudança conceitual $(A)$.

\section{Centralização do professor}

Por Centralização do professor entende-se o ensino centrado no professor, focado na transmissão de conhecimento. Quanto mais forte é essa característica, mais o professor é transmissor e menos agente de mudança conceitual, afastando-se do preconizado para o novo método de ensino-aprendizagem, centrado no estudante e não no professor (COSTA MCG, et al., 2016). Nesse aspecto espera-se, portanto, que os professores sejam agentes de mudança conceitual ao centralizar o ensino no aluno e não neles mesmos, como é na transmissão passiva de conhecimento.

Para a escala Centralização do Professor, observa-se que $75,36 \%$ dos respondentes foram classificados como agente de mudança conceitual, $18,84 \%$ classificados como facilitadores, $4,35 \%$ da amostra como organizadores e apenas 1 respondente classificado como transmissor. Dentre os gestores, observou-se que cinco foram classificados como agentes de mudança conceitual e dois como facilitadores (Tabela 4).

Tabela 4 - Perfil docente conforme Centralização do Professor.

\begin{tabular}{ccc}
\hline Categorias & Frequência & Percentual \\
\hline Agente de mudança conceitual & 52 & $75,36 \%$ \\
Facilitador & 13 & $18,84 \%$ \\
Organizador & 3 & $4,35 \%$ \\
Transmissor & 1 & $1,45 \%$ \\
\hline Total & $\mathbf{6 9}$ & $\mathbf{1 0 0} \%$ \\
\hline Fonte: SANTANA KR e CESARIO RR, 2020.
\end{tabular}

Este resultado sugere que o corpo docente avaliado é majoritariamente centrado no estudante, demonstrando uma alta proximidade com o recomendado pelas DCN que diz (BRASIL, 2014):

"Art. 26. O Curso de Graduação em Medicina terá projeto pedagógico centrado no aluno como sujeito da aprendizagem e apoiado no professor como facilitador e mediador do processo."

Um estudo avaliou aceitação, comportamento e aprendizagem de alunos do quarto ano de Ginecologia em relação a aulas centradas no professor (ACP) e aulas centradas nos alunos (ACA). Os alunos foram divididos em grupos, sendo que alguns receberam aulas expositivas (ACP) e alguns trabalharam os mesmos temas com aulas do tipo seminário e tutoria (ACA). Embora tenha-se percebido maior interação nas ACA e essa modalidade de aulas seja uma recomendação das DCN, os alunos participantes deste estudo demonstraram maior resistência à essa proposta.

$\mathrm{O}$ artigo ressalta que alunos que têm mais experiência em aulas centradas no professor tendem a rejeitar as atividades centradas no estudante e recomenda que talvez 0 indicado seriam inovações mescladas a modelos mais tradicionais, em especial se os professores não estiverem adequadamente treinados ou motivados para a mudança.

Concluiu-se que não é que as aulas centradas nos alunos sejam inviáveis em escolas médicas tradicionais, mas reforçam muito a necessidade de planejamento e capacitação docente para as mudanças (DEUS JM, et al., 2014). 


\section{Apreciação de Metodologias Ativas}

Por apreciação de Metodologias Ativas o Colt entende que quanto mais forte é essa característica, mais afinidade pelo método o professor tem, ou seja, mais próximo a agente de mudança conceitual ele está e, por conseguinte, mais distante de transmissor.

Para esta escala observa-se que $59,42 \%$ dos respondentes foram classificados como agente de mudança conceitual, $21,74 \%$ classificados como facilitadores, $15,94 \%$ da amostra como organizadores e apenas 2 respondentes, equivalente a $2,90 \%$, classificados como transmissores. Dentre os gestores, observou-se que seis foram classificados como agentes de mudança conceitual e um como facilitador (Tabela 5). As DCN em vigor falam sobre o uso de metodologias principalmente nos artigos 29 e 32 (BRASIL, 2014):

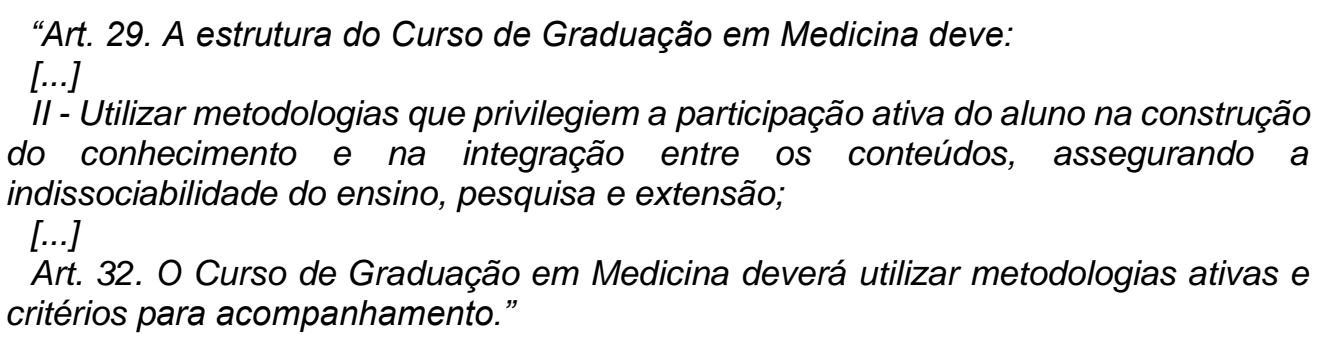

Tabela 5 - Perfil docente conforme Apreciação das Metodologias Ativas.

\begin{tabular}{ccc}
\hline Categorias & Frequência & Percentual \\
\hline Agente de mudança conceitual & 41 & $59,42 \%$ \\
Facilitador & 15 & $21,74 \%$ \\
Organizador & 11 & $15,94 \%$ \\
Transmissor & 2 & $2,90 \%$ \\
\hline Total & $\mathbf{6 9}$ & $\mathbf{1 0 0} \%$ \\
\hline
\end{tabular}

Fonte: Santana KR e Cesario RR, 2020.

É um grande desafio para professores formados no sistema tradicional, como é o caso de $89,86 \%$ da nossa amostra, alterar seu perfil de transmissor para agente de mudança conceitual, usando em sua prática esse tipo de metodologia.

Assim, consideramos alto nosso resultado de $81 \%$ de adequação do perfil docente, mas reconhecemos que $19 \%$ do corpo docente encontram-se ainda com perfil inadequado para a apreciação de metodologias ativas de ensino-aprendizagem e $41 \%$ do total ainda não alcançaram o grau máximo na escala, padrão-ouro para a mudança conceitual requerida para a transformação do profissional médico em agente (SEN, 2010).

Nagib LRC e Silva DM (2020) realizaram um estudo semelhante para identificar e analisar a relação entre a adoção de metodologias ativas no ensino de graduação em Ciências Contábeis. Como instrumento de pesquisa eles utilizaram um questionário com 13 situações hipotéticas vivenciadas em sala de aula em que 0 docente deveria apontar se adota ou não a metodologia demonstrada, por meio de uma nota, considerando uma escala de 0 a 10.

Ao final, concluíram que a adoção das categorias de metodologias ativas por professores do ensino de graduação em Ciências Contábeis no Brasil ainda era baixa e perceberam que a aula expositiva tradicional era uma das estratégias mais adotadas pelos professores pesquisados. No estudo, eles perceberam que à medida que o professor avançava nas fases do ciclo de vida docente, maior era a adoção das metodologias ativas e menor era o uso do método tradicional, sugerindo assim que o tempo de docência levaria o docente a alterar sua prática do modelo transmissor para o agente de mudança conceitual.

\section{Orientação para a Prática Profissional}

A Orientação para a Prática Profissional mede a capacidade do docente articular o ensino com a prática profissional futura do estudante. Neste aspecto, quanto menos habilidade de orientar para a prática mais transmissor é um professor e quanto mais ele melhora essa característica mais se aproxima do perfil de agente de mudança conceitual. 
Neste estudo, 44,93\% dos respondentes foram classificados como agente de mudança conceitual, $37,68 \%$ classificados como facilitadores, $13,04 \%$ da amostra como organizadores e apenas 3 respondentes, equivalente a $4,35 \%$, classificados como transmissores. Dentre os gestores, observou-se que cinco foram classificados como agentes de mudança conceitual e dois como facilitadores (Tabela 6).

Tabela 6 - Perfil docente conforme Orientação para a prática profissional.

\begin{tabular}{ccc}
\hline Categorias & Frequência & Percentual \\
\hline Agente de mudança conceitual & 31 & $44,93 \%$ \\
Facilitador & 26 & $37,68 \%$ \\
Organizador & 9 & $13,04 \%$ \\
Transmissor & 3 & $4,35 \%$ \\
\hline Total & $\mathbf{6 9}$ & $\mathbf{1 0 0} \%$
\end{tabular}

Fonte: Santana KR e Cesario RR, 2020.

As DCNs entendem que a competência para a prática profissional é compreendida como a capacidade de mobilizar conhecimentos, habilidades e atitudes capazes de solucionar os desafios que se apresentarão na vida profissional do estudante. Como dito no parágrafo único do artigo 8:

"Art. $8^{\circ}$ Para permitir a transformação das Diretrizes previstas no Capítulo I e os componentes curriculares contidos no Capítulo III ...

Parágrafo único. Para os efeitos desta Resolução, competência é compreendida como a capacidade de mobilizar conhecimentos, habilidades e atitudes, com utilização dos recursos disponíveis, e exprimindo-se em iniciativas e ações que traduzem desempenhos capazes de solucionar, com pertinência, oportunidade e sucesso, os desafios que se apresentam à prática profissional, em diferentes contextos do trabalho em saúde, traduzindo a excelência da prática médica, prioritariamente nos cenários do Sistema Único de Saúde (SUS)."

Assim, de acordo com o perfil exigido pelas DCNs, encontramos um resultado de $83 \%$ dos docentes com boa orientação para a prática profissional, um valor considerável para um curso ainda em seu $5^{\circ}$ ano de construção. Entretanto, $55 \%$ da amostra ainda não alcançou o padrão-ouro de agente de mudança conceitual. Esse dado é importante porque a orientação para prática é importantíssima para o desenvolvimento de um médico voltado para a clínica ampliada, na perspectiva da integralidade, algo defendido por nós como importante para desenvolver o sistema de saúde almejado pela Constituição (BRASIL, 1988).

O COLT, instrumento utilizado para explorar as concepções conscientes e inconscientes de aprendizado e ensino dos entrevistados, é auto-respondido, o que pode resultar em limitações pelo efeito da desejabilidade social e tendência a ter resultados tendenciosos, mas também em benefícios, por fornecerem feedbacks para a pessoa que se auto-avalia (EDWARDS AL, 1957; TELAU R, 2015; TAYLOR SN, 2014).

\section{CONSIDERAÇÕES FINAIS}

Com objetivo de caracterizar o corpo docente de um curso de medicina e suas concepções de ensinoaprendizagem em relação às DCN, este estudo encontrou um corpo docente com concepções de ensinoaprendizagem relativas a centralização do professor, apreciação de metodologias ativas e orientação para prática profissional adequadas ao perfil exigido pelas DCN, embora ainda sejam necessárias mais capacitações para torná-lo mais agente de mudança conceitual. Entende-se que tornar-se professor e tornarse médico são processos complexos, trata-se de construções das quais participam vários vetores pessoais, não apenas aqueles trabalhados durante a graduação ou pós-graduação. Por isso a docência, como a pesquisa e a prática de qualquer profissão, demanda capacitação específica. Em um contexto de transição paradigmática, faz-se necessário incentivar que os professores se envolvam sempre com o desenvolvimento docente, na missão de formar profissionais que contribuam para a reorientação do sistema de saúde. $O$ professor melhor habilitado para isso é o agente de mudança conceitual, perfil representado pela maioria dos professores pesquisados neste estudo. 


\section{REFERÊNCIAS}

1. BERBEL NN. A Problematização e a Aprendizagem Baseada em Problemas: Diferentes Termos ou Diferentes Caminhos? Interface (Botucatu), 1998; 2(2):139-154.

2. BRASIL. Constituição da República Federativa do Brasil. Brasília, DF, Senado, 1988.

3. BRASIL. Ministério da Educação. Resolução no 3, de 20 de junho de 2014. Institui Diretrizes Curriculares Nacionais do Curso de Graduação em Medicina e dá outras providências. Diário Oficial da União, Brasília, 23 de junho de 2014 - Seção 1 - pag. 8-11.

4. CARABETTA JV. Metodologia ativa na educação médica. Rev Med (São Paulo). 2016; 95(3):113-21.

5. CESARIO RR, et al. Alvorecer do Paradigma Sistêmico na Educação Médica. Rev. bras. educ. med., 2019; 43(supl. 1):305-313.

6. COSTA NMSC. Formação pedagógica de professores de medicina. Rev. Latino-Am. Enfermagem, Ribeirão Preto, 2010; 18(1):102-108.

7. COSTA MCG, et al. Ensino e Aprendizagem da Prática Profissional: Perspectiva de Estudantes de Medicina. Rev. bras. educ. med., 2016; 40(2): 245-253.

8. DEUS JM, et al. Aula centrada no aluno versus aula centrada no professor: desafios para mudança. Rev. bras. educ. med., 2014; 38(4):419-426.

9. EDWARDS AL. The social desirability variable in personality assessment and research. Dryden Press. 1957.

10. GARCIA MAA, SILVA ALB. Um perfil do docente de medicina e sua participação na reestruturação curricular. Rev. bras. educ. med., 2011; 35(1):58-68.

11. GOMES AP, REGO S. Transformação da educação médica: é possível formar um novo médico a partir de mudanças no método de ensino-aprendizagem? Rev. bras. educ. med., 2011; 35(4):557-566.

12. JACOBS JC, et al. Development of an instrument (the COLT) to measure conceptions on learning and teaching of teachers, in student-centred medical education. Med Teach, 2012; 34:e483-e491.

13. JACOBS JC, et al. Five teacher profiles in student-centred curricul based on their conceptions of learning and teaching. BMC Med Educ., 2014; 14:220.

14. LEITE VT, et al. Avaliação do Perfil dos Professores de Medicina de uma Universidade do Interior de Minas Gerais. Rev. bras. educ. med., 2020; 44(3):e096.

15. NAGIB LRC, SILVA DM. Adoção de metodologias ativas e sua relação com o ciclo de vida e a qualificação docente no ensino de graduação em ciências contábeis. Rev. contab. finanç., 2020; 31(82):145-164.

16. PAIVA VLMO. A linguagem dos emojis. Trab. linguist. apl., 2016; 55(2):379-401.

17. PERIM GL, et al. Desenvolvimento docente e a formação de médicos. Rev. bras. educ. med., 2009; 33(supl. 1):70-82.

18. PORTER C. Hot-Dog Fan Campaigns for New Emoji: popular texting characters include ice cream, pizza and sushi, but lack other pictures. Wall Street Journal, 2014. Disponível em https://www.wsj.com/articles/hot-dog-fancampaigns-for-new-emoji-1395801860. Acesso em 15/01/2020.

19. SEN, Amartya. Desenvolvimento como Liberdade. São Paulo: Companhia das Letras, 2010; 462p.

20. SILVA VO, SANTANA PMMA. Conteúdos curriculares e o Sistema Único de Saúde (SUS): categorias analíticas, lacunas e desafios. Interface (Botucatu), 2015; 19(52):121-132.

21. TAYLOR SN. Student self-assessment and multisource feedback assessment: exploring benefits, limitations, and remedies. Journal of Management Education, 2014; 38(3):359-383.

22. TELAU R. Ensinar - incentivar - mediar: dilemas nas formas de sentir, pensar e agir dos educadores dos CEFFAs sobre os processos de ensino/aprendizagem. Dissertação (Mestrado em Educação) - Universidade Federal de Minas Gerais, Belo Horizonte, 2015; 179p. 